\title{
Effect of hydrogen plasma treatment on the growth and microstructures of multiwalled carbon nanotubes
}

\section{S. K. Srivastava ${ }^{1,2,}$, V. D. Vankar ${ }^{1}$ and V. Kumar ${ }^{1,2}$}

The effect of hydrogen plasma treatment of iron oxide films on the growth and microstructure of carbon nanotubes (CNTs) by microwave plasma enhanced chemical vapor deposition process has been investigated. Microwave plasma was characterized in-situ using optical emission spectrometer. Morphology of the films was examined by scanning electron microscopy. Structural analysis was carried out by high resolution transmission electron microscopy (HRTEM) equipped with energy dispersive X-ray spectroscopy (EDS) and micro-diffraction attachments. It is found that oxide films without $\mathrm{H}_{2}$ plasma pretreatment or treated for lesser time resulted in CNT films with high percentage of carbonaceous particles and with embedded particles/nanorods distributed discontinuously in the cavity of the nanotubes. The embedded particles were found to be of iron carbide (Fe-C) as confirmed by HRTEM, EDS and micro-diffraction analysis. Experimental observations suggested that the iron oxide particles had poor catalytic action for CNT growth and in-situ reduction of oxide clusters to Fe by hydrogen plasma plays a key role in discontinuous filling of the nanotubes by the catalytic particles.

Keywords: Carbon nanotubes; Chemical vapor deposition; Iron carbide; MPECVD

Citation: S. K. Srivastava, V. D. Vankar and V. Kumar, "Effect of hydrogen plasma treatment on the growth and microstructures of multiwalled carbon nanotubes", Nano-Micro Lett. 2, 42-48 (2010). doi: 10.5101/nml.v2i1.p42-48

Since its discovery carbon nanotube [1] has attracted great attention because of its unique structure and properties. The unique structure-properties combination of CNTs have open up a wide range of research and their potential applications such as flat panel displays [2], gas storage [3], sensors [4], scanning probe microscope tips [5], single molecular transistors [6], nanotweezers [7], high power electrochemical capacitors [8], solar cells [9], etc. Several methods such as arc discharge [10], laser ablation [11], and different forms of chemical vapor deposition (CVD) [12-14] have been used to synthesize CNTs. CVD methods (both thermal and plasma enhanced) are reliable in producing multiwalled CNTs (MWNTs) with proper control over the process parameters. Plasma enhanced CVD, however, can produce CNTs with a higher growth rate, at low temperature, and with better reproducibility. The plasma not only ionizes the gas but also causes a local surface heating [15]. Consequently, growth temperature could be significantly decreased compared to non-plasma CVD processes. In addition, the plasma atmosphere may also influence the detailed catalyst surface kinetics in many ways [16] and hence the growth of carbon nanostructures.

Growth of CNTs and their microstructures depend on several parameters such as growth technique, feed gases, growth temperature, catalyst, and catalyst preparation method as well as their state (solid, liquid or vapor). In plasma assisted CVD processes, dilution gases play a critical role in the growth of CNTs and the structure of CNTs can be tailored by judicious control over gas composition [17]. In our earlier report, we investigated the effect of different dilution gases such as $\mathrm{H}_{2}, \mathrm{NH}_{3}$ and $\mathrm{N}_{2}$ and their different composition on the growth and microstructure of CNTs by microwave plasma enhanced CVD (MPECVD) process on Fe coated $\mathrm{Si}$ substrates using $\mathrm{C}_{2} \mathrm{H}_{2}$ as feed gas [17]. Plasma pretreatment of catalyst film can also affect the growth and structure of CNTs significantly especially in a high frequency plasma process such as MPECVD. To the best of our knowledge, effect of $\mathrm{H}_{2}$ plasma pretreatment of iron oxide films on the growth and structure of CNTs by MPECVD process have not been reported. Therefore, the motivation for the present

\footnotetext{
1 Department of Physics, Indian Institute of Technology Delhi, Hauz Khas, New Delhi-110016, India

2 National Physical Laboratory, Dr. K. S. Krishnan Marg, Pusa, New Delhi-110012, India

*Corresponding author. Email: srivassk@nplindia.org; Tel.: +91-11-45608617; Fax: +91-11-45609310
} 
study was to investigate the effect of $\mathrm{H}_{2}$ plasma treatment of iron oxide films on the growth and microstructure of CNTs by MPECVD technique. It is found that oxide films are relatively less catalytically active for CNTs growth. Further, oxide films treated for short duration by $\mathrm{H}_{2}$ plasma promote the formation of discontinuous metal filling of MWNTs compared to ones treated for longer duration or pure Fe films. Gradual in-situ reduction of oxide clusters to $\mathrm{Fe}$ by active hydrogen species of the plasma is suggested to be responsible for metal inclusion in nanotube cavity.

\section{Experimental details}

CNT films were deposited by MPECVD technique. The details of the experimental setup are described elsewhere [18]. In this study, oxidized Fe film of $\sim 10 \mathrm{~nm}$ thickness on Si-substrates was used as a catalyst. The catalyst coated substrate was loaded into the reactor chamber of the MPECVD system and the chamber was evacuated to a base pressure of $5.0 \times 10^{-3}$ Torr by a mechanical rotary pump. The reactor chamber was then purged many times by $\mathrm{H}_{2}$ gas to remove any residual gas. Finally, a pressure of 5 Torr for a $\mathrm{H}_{2} / \mathrm{Ar}$ flow rate of $50 \mathrm{sccm}$ was maintained with the help of a throttle valve. The plasma of $\mathrm{H}_{2}$ or $\mathrm{Ar}$ gas was ignited in the reactor chamber by applying microwave power. Three sets of samples (named as A, B, and C) were prepared. The experimental parameters for these samples are summarized in tab. 1. The growth of CNTs films by MPECVD process consisted of mainly two steps: (i) pretreatment of oxidized Fe films in $\mathrm{H}_{2}$ or Ar plasma for 5 15 min, (ii) introduction of $\mathrm{C}_{2} \mathrm{H}_{2}$ for the growth of CNTs films in $\mathrm{C}_{2} \mathrm{H}_{2}+\mathrm{H}_{2}$ gas mixture for $10 \mathrm{~min}$. Microwave power and chamber pressure of $500 \mathrm{~W}$ and 5 Torr respectively, were kept constant during both the pretreatment and growth for all the samples. Under these experimental conditions substrate temperature of $\sim 600{ }^{\circ} \mathrm{C}$ was estimated. Surface morphology of the as grown films was investigated by scanning electron microscopy (SEM, LEO $435 \mathrm{VP}$ ) operating at $15 \mathrm{kV}$. Microstructural analysis was carried out by transmission electron microscopy (TEM, Philips CM 12 and TECNAI 20T G2, FEI, Holland) equipped with micro-diffraction attachment. Energy dispersive x-ray spectroscopy (EDS, EDAX Inc., USA) attached with analytical TEM TECNAI 20T, G2 was used for elemental analysis of the embedded particles. The EDS spectra were recorded at an operating voltage of $200 \mathrm{kV}$, probe size of $15 \mathrm{~nm}$ using low background double tilt holder and GENESIS software. For TEM specimens, a piece of film was peeled-off from the substrate and ultrasonicated in acetone for $10 \mathrm{~min}$. Few drops of the dispersion were then placed on holy carbon coated copper grid.

In-situ diagnosis of microwave plasma was carried out using an optical emission spectrometer (OES) (EP200 Msd,
Verity Instrument). This instrument consists of a scanning monochromator with a focal length of $0.2 \mathrm{~m}$ and a spectral range of 200 900 nm. A concave holographic grating with an optimal resolution of $0.2 \mathrm{~nm}$ and a slit size of $50 \mu \mathrm{m}$ was used. The detector was a photomultiplier tube. The radiation from the plasma was collected through a quartz window and focused onto the monochromator slit by a quartz lens. The acquisition and analysis were done by a computer. The plasma was recorded at the centre of the cavity where both electron temperature and plasma density were the maximum.

\section{Results and discussion}

Figure 1(a)-(c) show the representative low magnified SEM images of samples A, B and C respectively. The corresponding highly magnified SEM images of these samples are shown in Fig. 1(d), 1(e) and 1(f) respectively. Sample A contains high percentage of carbonaceous clusters and CNTs appear to be embedded in amorphous carbon (a-C) matrix whereas sample B has high density of entangled and relatively clean nanotubes though it also has a lot of carbonaceous clusters. Sample $\mathrm{C}$ has the highest density of clean nanotubes among the three samples.

TEM studies showed that all the samples had MWNTs with large variation in their length and diameters. Inner and outer diameters of the nanotubes were found in the range of $5 \sim 15 \mathrm{~nm}$ and 10 50 $\mathrm{nm}$ respectively. However, a remarkable change in the microstructures of CNTs of the three samples was observed. Samples A and B were observed to contain $\sim 50 \%$ of nanotubes with embedded catalytic particles in their cavities (estimated from TEM investigations). Some of the representative TEM images of the filled MWNTs of sample B (and sample A) are shown in Fig. 2. A low magnification TEM image of the filled CNT is shown in Fig. 2 (a) showing embedded particles in different sections of the tube. The magnified images of the filled regions are shown in the insets of Fig. 2 (a). For more clarity of nanotube filling, a dark field image of such a nanotube is shown in Fig. 2 (b). The embedded particles are elongated along the tube axis forming nanorods. The length of such nanorods was found in the range of 30-200 $\mathrm{nm}$ and diameter equal to inner diameter of the CNTs. Some of the embedded nanorods were more than $500 \mathrm{~nm}$ in length (see Fig. 2 (d)). The shape and size of the embedded particles and nanotube cores were found to be interlinked. It is to be noticed that embedded particles/nanorods are lying mostly at positions where either tube is distorted or compartment formation occurs as clearly seen from TEM images in Fig. 2 (b) and (c). TEM image shown in Fig. 2 (d) clearly suggests the fluid-like nature of the embedded particles and the inner wall of the tube is completely wet by the embedded particles. The typical HRTEM image of an embedded particle is shown in Fig. 2(e) which clearly shows that the particles have crystalline structure. The inter-planar spacing of $\sim 0.238 \mathrm{~nm}$ and

Table 1: Experimental parameters during catalyst pretreatment and CNTs growth.

\begin{tabular}{cccccc}
\hline \multirow{2}{*}{ Sample } & \multirow{2}{*}{ Catalyst } & \multicolumn{3}{c}{ During Pretreatment } & \multicolumn{2}{c}{ During Growth } \\
\cline { 3 - 5 } & & Gas & Time (min.) & Gas Flow (sccm) & Gas composition \\
\hline A & Oxidized Fe film & $\mathrm{Ar}$ & 5 & 50 & $\mathrm{C}_{2} \mathrm{H}_{2} / \mathrm{H}_{2}$ \\
B & Oxidized Fe film & $\mathrm{H}_{2}$ & 5 & 50 & $\mathrm{C}_{2} \mathrm{H}_{2} / \mathrm{H}_{2}$ \\
C & Oxidized Fe film & $\mathrm{H}_{2}$ & 15 & 50 & $\mathrm{C}_{2} \mathrm{H}_{2} / \mathrm{H}_{2}$ \\
\hline
\end{tabular}



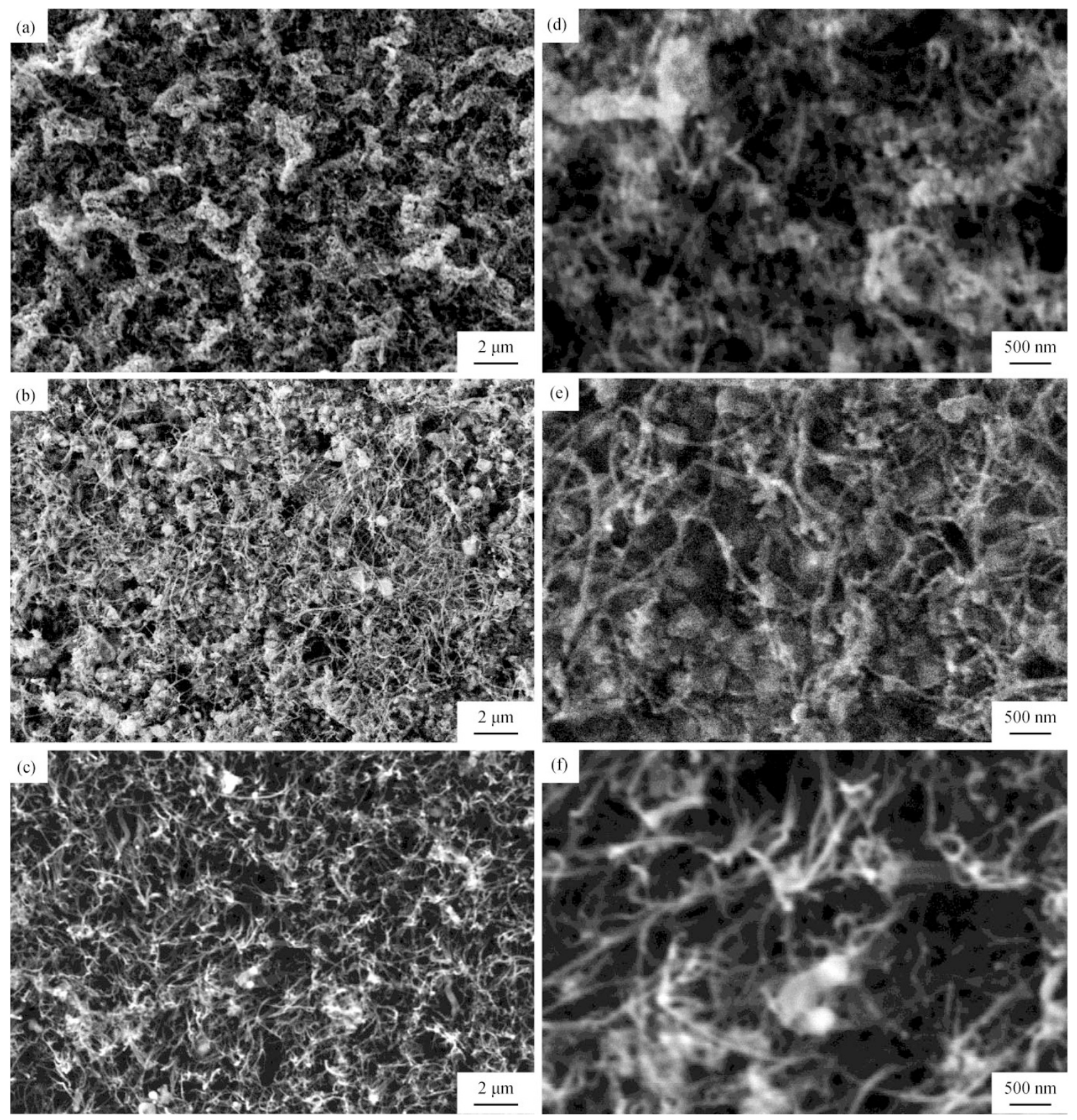

FIG. 1. SEM micrographs of CNT samples (a) A, (b) B, and (c) C. Corresponding highly magnified SEM images of samples A, B and C are shown in (d), (e) and (f) respectively.

0.201 correspond to that of iron carbide. Therefore, the embedded particles/nanorods are of iron carbide. The selected area micro-diffraction pattern of an embedded particle is also shown in the inset of Fig. 2 (c) also confirming that the particles are crystalline $\mathrm{Fe}_{3} \mathrm{C}$. The measured d- values $0.215 \mathrm{~nm}, 0.154 \mathrm{~nm}$ and $0.210 \mathrm{~nm}$ match with the theoretical values of (102), (221) and (12-1) planes of orthorhombic $\mathrm{Fe}_{3} \mathrm{C}$ phase within limits of measurement errors. The measured angles between the planes (102) and (221); (22-1) and (12-1); (12-1) and (102) are $48^{\circ}, 47^{\circ}$ and $95^{\circ}$ respectively which are quite comparable with their theoretical values.

Figure 3(a) shows the EDS spectrum recorded from embedded particle region of the tube (shown in the inset). Strong signals corresponding to $\mathrm{Fe}\left(6.400 \mathrm{keV}\right.$ for $\mathrm{K}_{\alpha}$ and $7.058 \mathrm{keV}$ for $\mathrm{K}_{\beta}$ ) are observed. Signals corresponding to $\mathrm{C}$ and $\mathrm{Cu}$ are attributed to carbon coated $\mathrm{Cu}$-microgrid used for specimen preparation. A noticeab small signal corresponding to $\mathrm{O}$ is also observed which could be due to oxidation of Fe particles lying on the outer surface of the tube since the probe used for recording spectra was coarse ( $>15 \mathrm{~nm}$ ) and also the specimen was exposed to the ambience for many days after preparation. Figure $3(\mathrm{~b})$ shows the representative TEM image of sample C. High percentages ( $>80 \%$ estimated from TEM investigation) of nanotubes were found to have hollow cavity (without embedded particles). However, some very random shaped fibers were also observed. 

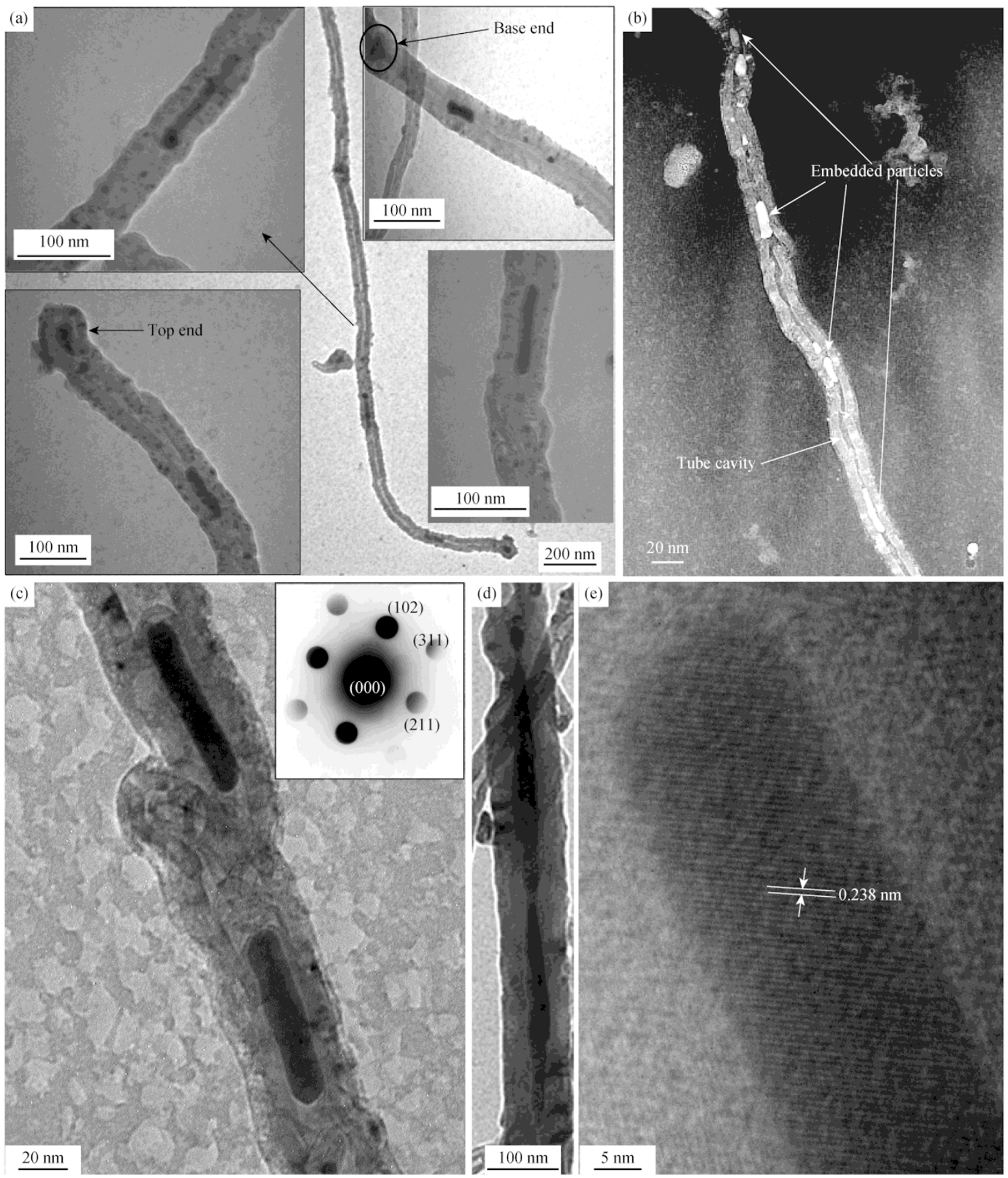

(e)

FIG. 2. TEM micrographs of sample A (or B) showing (a) low magnification view of embedded particles in the cavity of the nanotube. Magnified view of different sections of the nanotube are shown in the inset, (b) dark field image of nanotube showing discontinuous filling throughout the tube, (c) elongated catalytic particles trapped in the nanotube cavity, (d) fluid-like nature of the embedded particles, (e) HRTEM micrograph of a representative embedded particle.

Several models proposed on the role of metal catalysts in the growth of CNTs by CVD methods mostly based on the mechanism suggested by Baker et al. [19]. In the present study, $\mathrm{Ar} / \mathrm{H}_{2}$ plasma pretreatment of the iron oxide film resulted into high density of nanoparticles (not shown here) which catalyzed the nucleation and growth of CNTs on the introduction of $\mathrm{C}_{2} \mathrm{H}_{2}$ in the plasma [17]. The observation of a-C in samples A, and $\mathrm{B}$ and relative increase in the percentage of a-C with decreasing $\mathrm{H}_{2}$ plasma pretreatment time of oxide films suggest the poor catalytic activity of oxide for CNTs growth as also reported by other methods [20,21]. In case of sample $C$, most of the iron oxide clusters are supposed to be reduced to Fe after 15 minutes of $\mathrm{H}_{2}$ plasma treatment since plasma contains a lot of active $\mathrm{H}$-species as observed by in-situ OES of the $\mathrm{H}_{2}$ and $\mathrm{H}_{2}+\mathrm{C}_{2} \mathrm{H}_{2}$ plasma in 200 900 $\mathrm{nm}$ wavelength range as shown in Fig. 4. The major emission lines observed in the $\mathrm{H}_{2}$ plasma are $\mathrm{H}_{\alpha}$ (656.6), 

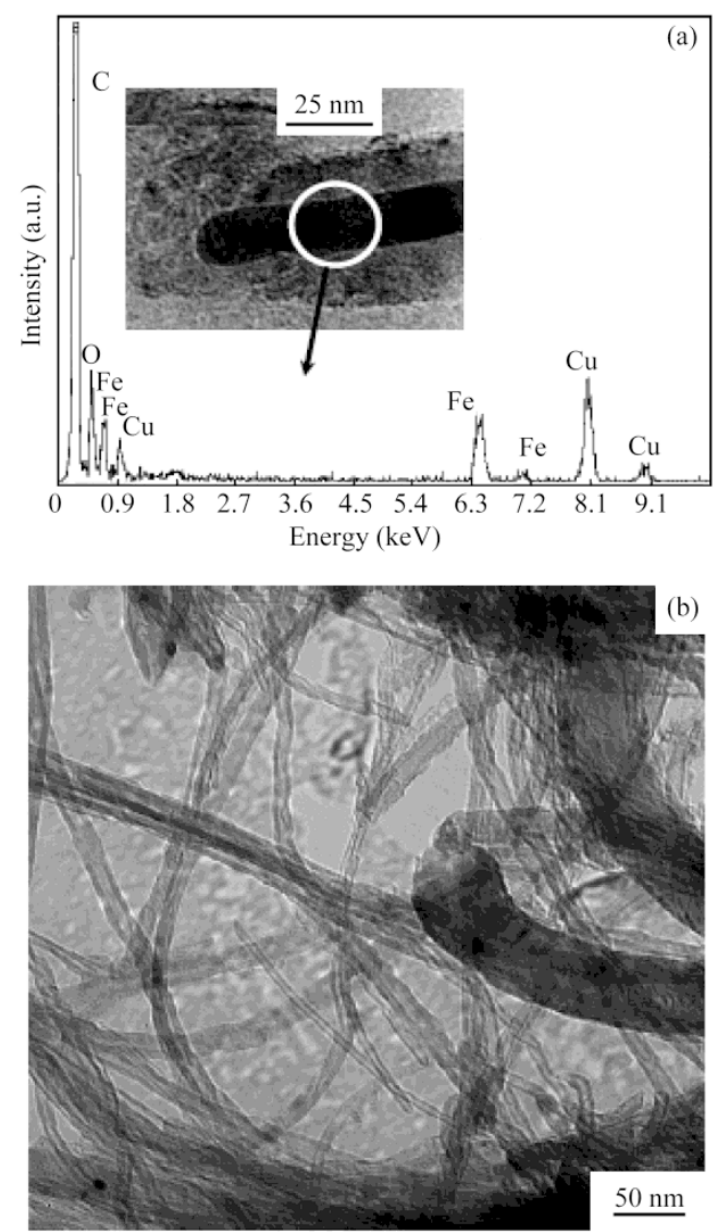

FIG. 3. (a) Typical EDS spectrum of the embedded particle. Corresponding embedded particle is shown in the inset, (b) typical TEM micrograph of sample $\mathrm{C}$ showing nanotubes without embedded particles in the cavity.

$\mathrm{H}_{\beta}$ (486.5), $\mathrm{H}_{\gamma}$ (434.3) and $\mathrm{H}_{2}$ band [22-24]. Upon $\mathrm{C}_{2} \mathrm{H}_{2}$ addition, apart from all lines and bands present in $\mathrm{H}_{2}$ plasma, $\mathrm{C}_{2}$ (516.5) Swan band and $\mathrm{CH}$ (431.4) band were also observed. But in the whole spectrum $\mathrm{H}$-species which play a significant role in removing the excess carbon species in CNTs nucleation and growth by forming volatile species [17] was the most intense whereas $\mathrm{C}_{2}$ and $\mathrm{CH}$ lines had very low intensity. Random growth orientation and the preferential precipitation of carbon around the particle might have incorporated defects in the growing nanotube walls and hence the irregular microstructures.

Although the $\mathrm{Fe} / \mathrm{Fe}-\mathrm{C}$ carbide filling of high percentage of CNTs in case of samples A and B is not yet fully understood, a possible mechanism on the basis of our experimental observations may be suggested as follows. Upon treatment in $\mathrm{H}_{2}$ plasma for 5 minutes or lesser times, iron oxides clusters possibly get reduced to Fe partly and become catalytically active. When $\mathrm{C}_{2} \mathrm{H}_{2}$ is introduced in the plasma, CNTs nucleate around the partly formed Fe particles as per the mechanism by Baker et al. [19]. Carbon species present in the plasma get adsorbed on the surface of the partly formed Fe particles followed by the diffusion of carbon atoms into/on the $\mathrm{Fe}$ particles to form metastable iron-carbon alloy, which finally precipitates as crystalline tubular structures upon supersaturation. The tube so formed continues to grow away from the particle by the

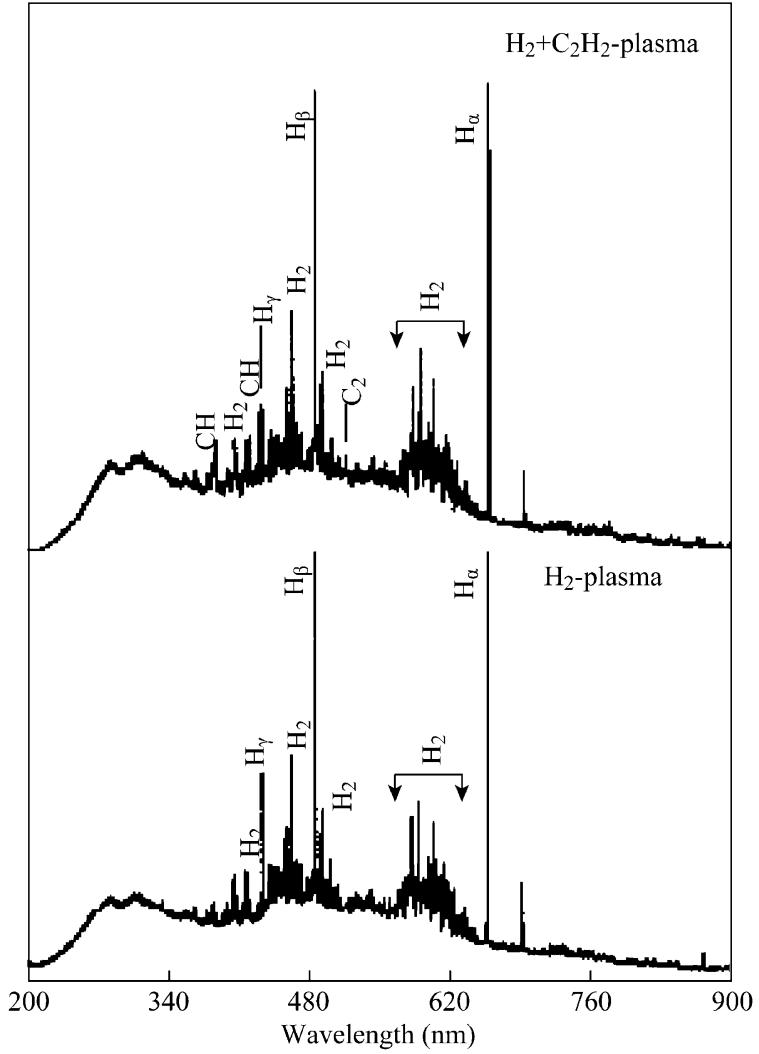

FIG. 4. OES spectra of $\mathrm{H}_{2}$ and $\mathrm{H}_{2}+\mathrm{C}_{2} \mathrm{H}_{2}$ microwave plasma showing different plasma species.

continuous supply of carbon atoms on the contact region between catalytic particle and the already formed tube segment. The precipitated graphite layers tend to wrap tightly around the Fe clusters to minimize their surface energy consequently changing the shape of the particles (as observed in Fig. 2). The growth of nanotubes and reduction of oxide particles to Fe occur simultaneously in $\mathrm{C}_{2} \mathrm{H}_{2}+\mathrm{H}_{2}$ plasma. As the reduction process of oxide clusters continues with time, more and more parts of the $\mathrm{Fe}$ particles participate in the growth process resulting in encapsulation of larger sections of the particle. The mechanism for discontinuous filling and rod formation can be explained as below.

It is known that the melting point of nanocrystalline particles is size dependent [25]. In addition, carbon-saturated metallic nanoparticles melt at temperatures far below the bulk melting point of the metal [26,27]. Schaper et al. [27] observed the motion of liquid/quasi liquid metal particle in the core of nanotube along the tube axis. They accounted this to the surface tensional forces buildup at the core material/tube interface and the compressive stress during thickness growth of the graphitic wall. Similarly, Ichihashi et al [28] observed tubulization of carbon nanopillar and liquid-like behavior of iron particles in real time TEM measurements of a-C nanopillars. In our observations also catalyst particles are supposed to be in the liquid or quasi-liquid state during nanotube growth (as observed in Fig. 2 (d)) because of the low dimension and the metal-carbon alloy formation. Also, it is believed that the local temperature 
could be much higher due to plasma [15] compared to the average substrate temperature measured $\left(\sim 600^{\circ} \mathrm{C}\right)$. The elongation or stretching of the encapsulated particle occurs probably due to the interplay between the catalyst surface and the graphite layers precipitated. The precipitated graphite layers have high surface and elastic energy and the layers tend to tighten the catalytic particle to acquire minimum energy configuration consequently moulding the embedded particle as per the shape of the inner core of the growing tube. Sometimes catalytic particles can even be stretched into parts and it is believed that the catalytic particles are fragmented at different stages of the growth irregularly. The smaller particles move in the core because of their liquid-like nature and coalesce to form bigger particle. Therefore, nanorods are formed as a result of frequent fragmentation of the catalytic particle during growth followed by their coalescence in the tube core. More and more parts of the catalyst particle are trapped into the tube as it grows longer and longer resulting in the discontinuous filling of the nanotubes (see Fig. 2). In MPECVD process, cooling (after growth process) takes place comparatively faster once plasma is switched off. Eventually some amount of carbon remains in the embedded particles even at room temperature resulting in carbide phase of these particles. Estimation of actual percentage of carbon in these particles is difficult at this stage as some of the embedded particles may be pure Fe.

\section{Conclusion}

The effect of hydrogen plasma pretreatment of iron oxide films on the growth and microstructure of CNTs has been investigated by MPECVD process. Experimental observations suggested that iron oxide itself has poor catalytic activity for CNTs growth. Hydrogen plasma plays an important role both in catalyst preparation and CNTs growth. Oxide films treated for lesser time result in $\mathrm{Fe} / \mathrm{Fe}-\mathrm{C}$ filled MWNTs which is attributed to in-situ reduction of iron oxide clusters to Fe by active hydrogen species in the plasma. Discontinuous filling has been suggested due to the fragmentation of the catalytic particle at different stage of growth and their liquid-like nature. The present results provide useful information for achieving metal/metal carbide filled CNTs using metal oxide as the catalyst.

The authors gratefully acknowledge to the electron microscopy division, All India Institute of Medical Sciences (AIIMS), New Delhi for SEM and Dr. D.V. Sridhara Rao, DMRL, Hyderabad for TEM analysis of the samples.

Received 4 March 2010; accepted 11 March 2010; published online 30 March 2010.

\section{References}

1. S. Iijima, Nature 354, 56 (1991). doi:10.1038/354056a0
2. S. J. Tans, A.R.M. Verschueren and C. Dekker, Nature 393, 49 (1998). doi:10.1038/29954

3. S. S. Wong, E. Joselevich, A. T. Woolley, C. L. Cheung and C. M. Lieber, Nature 394, 52 (1998). doi:10.1038/27873

4. C. Liu, Y. Y. Fan, M. Liu, H. T. Cong, H. M. Cheng and M. S. Dresselhaus, Science 286, 1127 (1999). doi:10.1126/ science.286.5442.1127

5. M. Meyyappan, Carbon Nanotubes: Science and Applications, CRC press, New York, 2004, p. 65. doi:10.12 $\underline{01 / 9780203494936}$

6. N. Grobert, W. K. Hsu, Y. Q. Zhu, J. P. Hare, H. W. Kroto, D. R. M. Walton, M. Terrones, H. Terrones, Ph. Redlich, M. Rühle, R. Escudero and F. Morales, Appl. Phys. Lett. 75, 3363 (1999). doi:10.1063/1.125352

7. J. Sloan, A.I. Hutchson and M. L. H. Green, Chem. Comm. 13, 1319 (2002). doi:10.1039/b200537a

8. E. Muñoz-Sandoval, F. López-Urías, A. Díaz-Ortiz, M. Terrones, M. Reyes-Reyes and J. L. Morán- López, J. Magn. Magn. Mater. 272-276 (2004) E1255. doi:10.1016/ j.jmmm.2003.12.1379

9. F. Geng and H. Cong, Physica B 382, 300 (2006). doi:10.1016/j.physb.2006.03.003

10. R. Sen, A. Govindaraj and C. N. R. Rao, Chem. Mater. 9, 2078 (1997). doi:10.1021/cm9700965

11. D.-C. Li, L. Dai, S. Huang, A. W. H. Mau and Z. L. Wang, Chem. Phys. Lett. 316, 349 (2000). doi:10.1016/S00092614(99)01334-2

12. X. Ma, Y. Cai, N. Lun, Q. Ao, S. Li, F. Li, and S. Wen, Mater. Lett. 57, 2879 (2003).

13. A. Leonhardt, M. Ritschel, R. Kozhuharova, A. Graff, T. Muhl, R. Hunle, I. Monch, D. Elefant and C. M. Schneider, Diamond Relat. Mater. 12, 790 (2003). doi:10.1016/ $\underline{\text { S0925-9635(02)00325-4 }}$

14. Z. J. Liu, R. Che, Z. Xu and L. M. Peng, Synthetic Met. 128, 191 (2002). doi:10.1016/S0379-6779(02)00005-X

15. K. B. K. Teo, D. B Hash, R. G. Lacerda, N. L. Rupesinghe, M. S. Bell, S. H. Dalal, D. Bose, T. R. Govindan, B. A. Cruden, M. Chhowalla, G. A. J. Amaratunga, M. Meyyappan and W. I. Milne, Nano Lett. 4, 921 (2004). doi: $10.1021 / \mathrm{nl} 049629 \mathrm{~g}$

16. D. B. Hash and M. Meyyappan, J. Appl. Phys. 93, 750 (2003). doi:10.1063/1.1525854

17. S. K. Srivastava, V. D. Vankar and V. Kumar, Thin Solid Films 515, 1552 (2006). doi:10.1016/j.tsf.2006.05.009

18. S. K. Srivastava, A. K. Shukla, V. D. Vankar and V. Kumar, Thin Solid Films 492, 124 (2005). doi:10.1016/j.tsf. 2005.07.283

19. R. T. K. Baker, and P. S. Harris, in: J.P.L. Walker and P.A. Thrower (Eds.), Chemistry and Physics of Carbon, Dekker, New York, 1978, p.83.

20. I. Wolf and H. J. Grabke, Solid State Commun. 54, 5 (1985). doi:10.1016/0038-1098(85)91021-X 
21. M. Perez-Cabero, I. Rodriguez-Ramos and A. GuerreroRuiz, J. Catal. 215, 305 (2003). doi:10.1016/S00219517(03)00026-5

22. R. W. B. Pearse and A. G. Gaydon, The Identification of Molecular Spectra, Chapman and Hall, London, 1976.

23. T. Vandevelde, M. Nesladek, C. Quaeyhaegens and L. Stals, Thin Solid Films 290-291, 143 (1996). doi:10.1016/ S0040-6090(96)09189-4

24. T. Vandevelde, T. D. Wu, C. Quaeyhaegens, J. Vlekken, M. D'Olieslaeger and L. Stals, Thin Solid Films 340, 159 (1999). doi:10.1016/S0040-6090(98)01410-2
25. P. A. Buffat, Thin Solid Films 32, 283 (1976). doi:10. $\underline{1016 / 0040-6090(76) 90315-1}$

26. Q. Jiang, N. Aya and F. G. Shi, Appl. Phys. A 64, 627 (1997). doi:10.1007/s003390050529

27. A. K. Schaper, H. Hou, A. Greiner and F. Phillipp, J. Catal. 222, 250 (2004). doi:10.1016/j.jcat.2003.11.011

28. T. Ichihashi, J. I. Fijita, M. Ishida and Y. Ochiai, Phys. Rev. Lett. 92, 215702 (2004). doi:10.1103/PhysRevLett.92. $\underline{215702}$ 